\title{
Finite-Temperature Phase Diagram of the Hubbard Model
}

\author{
Gabriele Migliorini ${ }^{1,2}$ and A. Nihat Berker ${ }^{1,2,3}$ \\ ${ }^{1}$ Department of Physics, Massachusetts Institute of Technology \\ Cambridge, Massachusetts 02139, U.S.A. \\ ${ }^{2}$ Feza Gürsey Research Center for Basic Sciences \\ Çengelköy, Istanbul 81220, TURKEY \\ ${ }^{3}$ Department of Physics, Istanbul Technical University \\ Maslak, Istanbul 80626, TURKEY
}

\begin{abstract}
The finite-temperature phase diagram of the Hubbard model in $d=3$ is obtained from renormalization-group analysis. It exhibits, around half filling, an antiferromagnetic phase and, between 30\%-40\% electron or hole doping from half filling, a new $\tau$ phase in which the electron hopping strength $t$ asymptotically becomes infinite under repeated rescalings. Next to the $\tau$ phase, a first-order phase boundary with very narrow phase separation (less than $2 \%$ jump in electron density) occurs. At temperatures above the $\tau$ phase, an incommensurate spin modulation phase is indicated. In $d=2$, we find that the Hubbard model has no phase transition at finite temperature.
\end{abstract}

The Hubbard model [1] is the bare-essentials realistic model of electronic conduction, yet essentially no knowledge has existed even phenomenologically on its most frontal macroscopic feature, namely its phase diagram at finite temperatures. In this research, we obtain a finite-temperature phase diagram for the Hubbard model in spatial dimension $d=3$, from an approximate renormalization-group calculation with flows in a 10-dimensional Hamiltonian space. This rich phase diagram, in the variables of temperature, electron density, and on-site repulsion, exhibits, around half-filling, an antiferromagnetic phase completely due to electron hopping. At 30-40\% electron or hole doping from half filling, a new $\tau$ phase occurs with distinctive conduction property. In the neighborhood of the $\tau$ phase, a phase separation so narrow that the jump in electron density is less than $2 \%$ occurs. At temperatures above the $\tau$ phase, an incommensurate frozen spin modulation phase is indicated. In $d=2$, no phase separation or other phase transition occurs at finite temperature in the Hubbard model, in contrast [2,3] to the closely related, but less realistic, tJ model of electronic conduction.

The Hubbard model is defined by the Hamiltonian

$$
\begin{aligned}
-\beta \mathcal{H}= & -t \sum_{<i j>, \sigma}\left(c_{i \sigma}^{\dagger} c_{j \sigma}+c_{j \sigma}^{\dagger} c_{i \sigma}\right) \\
& -U \sum_{i} n_{i \uparrow} n_{i \downarrow}+\mu \sum_{i} n_{i},
\end{aligned}
$$

where $c_{i \sigma}^{\dagger}$ and $c_{i \sigma}$ are the electron creation and annihilation operators with spin $\sigma=\uparrow$ or $\downarrow$ at site $i$ of a cubic lattice, $\langle i j\rangle$ indicates summation over all nearestneighbor pairs of sites, and

$$
n_{i \sigma}=c_{i \sigma}^{\dagger} c_{i \sigma} \text { and } n_{i}=n_{i \uparrow}+n_{i \downarrow}
$$

are the electron number operators. The terms in the Hamiltonian of Eq.(1) are, respectively, the kinetic energy term, the on-site repulsion $(U>0)$ term, and the 
chemical potential term included in order to study the system over its entire density range from zero to two electrons per site.

The renormalization-group transformation is formulated [3: [4] by first considering a $d=1$ system. An exact renormalization group transformation can be formally written,

$<u_{1} u_{3} u_{5} \ldots\left|e^{-\beta^{\prime} \mathcal{H}^{\prime}}\right| v_{1} v_{3} v_{5} \ldots>$
$\sum_{w_{2} w_{4} w_{6} \ldots}<u_{1} w_{2} u_{3} w_{4} u_{5} w_{6} \ldots\left|e^{-\beta \mathcal{H}}\right| v_{1} w_{2} v_{3} w_{4} v_{5} w_{6} \ldots>$,

where $u_{1}, w_{2}, v_{3}$, etc. represent the single-site states. Primes indicate the renormalized system. The transformation given in Eq.(3) conserves the partition function, $Z=Z^{\prime}$, but cannot be implemented due to the noncommutativity of the operators in the Hamiltonian. An approximation is used:

$$
\begin{aligned}
\operatorname{Tr}_{\text {even sites }} \exp (-\beta \mathcal{H})= & \operatorname{Tr}_{\text {even sites }} \exp \left(\sum_{i}^{\text {even }}-\beta \mathcal{H}(i-1, i)-\beta \mathcal{H}(i, i+1)\right) \simeq \prod_{i}^{\text {even }} \operatorname{Tr}_{w_{i}} \exp (-\beta \mathcal{H}(i-1, i)-\beta \mathcal{H}(i, i+1)) \\
& =\prod_{i}^{\text {even }} \exp \left(-\beta^{\prime} \mathcal{H}^{\prime}(i-1, i+1)\right) \simeq \exp \left(\sum_{i}^{\text {even }}-\beta^{\prime} \mathcal{H}^{\prime}(i-1, i+1)\right)=\exp \left(-\beta^{\prime} \mathcal{H}^{\prime}\right),
\end{aligned}
$$

where

$$
\begin{aligned}
& -\beta \mathcal{H}(i, j)=-t\left(c_{i \sigma}^{\dagger} c_{j \sigma}+c_{j \sigma}^{\dagger} c_{i \sigma}\right) \\
& -(U / 2 d) \sum_{i}\left(n_{i \uparrow} n_{i \downarrow}+n_{j \uparrow} n_{j \downarrow}\right)+(\mu / 2 d) \sum_{i}\left(n_{i}+n_{j}\right) .
\end{aligned}
$$

Thus, the approximation consists in neglecting the commutation relations beyond segments of three consecutive unrenormalized sites. This approximation is effected twice $(\simeq)$ in Eq.(4), in opposing directions, hopefully with compensatory effect. The crux of the calculation is extracted from the third step in Eq.(4),

$$
\operatorname{Tr}_{w_{2}} e^{-\beta \mathcal{H}(1,2)-\beta \mathcal{H}(2,3)}=e^{-\beta^{\prime} \mathcal{H}^{\prime}(1,3)} .
$$

When written in terms of three-site (on the left) and two-site (on the right) matrix elements, this equation amounts to contracting a $64 \times 64$ matrix into a $16 \times 16$ matrix. This operation is facilitated by block diagonalization of the matrices, using the conservations of particles, total spin magnitude, total spin $z$-component, and parity, so that the largest blocks are $4 \times 4$ and $2 \times 2$ for the unrenormalized and renormalized systems, respectively. Thus, a renormalized Hamiltonian $-\beta^{\prime} \mathcal{H}^{\prime}$ is extracted. The closed form of $-\beta^{\prime} \mathcal{H}^{\prime}$ is more general than Eq.(1), namely

$$
\begin{aligned}
-\beta \mathcal{H}= & -\sum_{<i j>, \sigma}\left[t_{0} h_{i-\sigma} h_{j-\sigma}+t_{1}\left(n_{i-\sigma} h_{j-\sigma}+h_{i-\sigma} n_{j-\sigma}\right)+t_{2} n_{i-\sigma} n_{j-\sigma}\right]\left(c_{i \sigma}^{\dagger} c_{j \sigma}+c_{j \sigma}^{\dagger} c_{i \sigma}\right) \\
& -t_{x} \sum_{<i j>}\left(c_{i \uparrow}^{\dagger} c_{j \uparrow} c_{i \downarrow}^{\dagger} c_{j \downarrow}+c_{j \uparrow}^{\dagger} c_{i \uparrow} c_{j \downarrow}^{\dagger} c_{i \downarrow}\right)-U \sum_{i} n_{i \uparrow} n_{i \downarrow}+\mu \sum_{i} n_{i} \\
& +\sum_{<i j>}\left[J \vec{s}_{i} \cdot \vec{s}_{j}+V_{2} n_{i} n_{j}+V_{3}\left(n_{i \uparrow} n_{i \downarrow} n_{j}+n_{i} n_{j \uparrow} n_{j \downarrow}\right)+V_{4} n_{i \uparrow} n_{i \downarrow} n_{j \uparrow} n_{j \downarrow}\right],
\end{aligned}
$$


where the hole operator is $h_{i \sigma} \equiv 1-n_{i \sigma}$ and the electron spin operator at site $i$ is

$$
\vec{s}_{i}=\sum_{\sigma, \sigma^{\prime}} c_{i \sigma}^{\dagger} \vec{s}_{\sigma \sigma^{\prime}} c_{i \sigma^{\prime}},
$$

where $\vec{s}_{\sigma \sigma^{\prime}}$ is the vector of Pauli spin matrices. The four hopping terms in the flow Hamiltonian [Eq.(7)] correspond to one electron hopping with or without the opposite spin electron present at the initial and final sites (two of these processes are related by hermitivity and therefore have the same hopping strength $t_{1}$ ) and to two electrons simultaneously hopping from one site to a neighboring site. These four processes can be called vacancy hopping $\left(t_{0}\right)$, pair breaking $\left(t_{1}\right)$, pair hopping $\left(t_{2}\right)$, and vacancypair interchange $\left(t_{x}\right)$. For

$$
t_{0}=t_{1}=t_{2}, \quad t_{x}=J=V_{2}=V_{3}=V_{4}=0,
$$

the flow Hamiltonian [Eq.(7)] reduces to the Hubbard Hamiltonian [Eq.(1)]. Thus, Eqs.(9) are the initial conditions of our renormalization-group flows. However, in general, the hopping strengths renormalize differently and the new interactions are generated under rescaling, so that the renormalization-group flows are in the 10dimensional, $\vec{K}=\left(t_{0}, t_{1}, t_{2}, t_{x}, U, \mu, J, V_{2}, V_{3}, V_{4}\right)$, Hamiltonian space.

The transformation is implemented in $d>1$ by using the Migdal-Kadanoff procedure, so that $\overrightarrow{K^{\prime}}=$ $\left(b^{d-1} / f\right) \vec{R}(f \vec{K})$ where $b=2$ is the length-rescaling factor, the function $\vec{R}$ is the contraction process specified in the previous paragraph, and $f$ is an arbitrary bondmoving factor, set to yield the correct transition temperature of the Ising model $(f=1.2279$ and 1.4024 in $d=3$ and 2). This renormalization-group transformation yields known information about quantum systems, such as, in $d=1$, the absence finite-temperature phase transitions; in $d=2$, a conventional phase transiton for the Ising model, a Kosterlitz-Thouless transition for the XY model [5.67, no phase transition for the Heisenberg model; in $d=3$, ferromagnetic and antiferromagnetic phase transitions for the Heisenberg model, the antiferromagnetic transition occurring at a $22 \%$ higher temperature than the ferromagnetic transition, a purely quantum mechanical effect [7]. The 10-dimensional renormalizationgroup flows also conserve the particle-hole symmetry, given the map:

$$
\begin{aligned}
\bar{t}_{0} & =t_{2}, \bar{t}_{1}=t_{1}, \bar{t}_{2}=t_{0}, \bar{t}_{x}=t_{x}, \bar{J}=J, \\
\bar{\mu} & =-\mu+U+2 d V_{3}-2 d V_{4}, \bar{U}=U+4 d V_{3}-2 d V_{4}, \\
\bar{V}_{2} & =V_{2}-2 V_{3}+V_{4}, \bar{V}_{3}=-V_{3}+V_{4}, \bar{V}_{4}=V_{4} .
\end{aligned}
$$

The global analysis of the renormalization-group flows yields the phase diagram of the system. We have thus obtained the global phase diagram of Hubbard model, presented here in Figures 1-3, where first- and secondorder phase boundaries are respectively shown by dottted and full curves. The particle-hole symmetry [Eq.(10)] 
dictates that the Hubbard model [Eq.(1)] phase diagrams be symmetric about $\mu / U=0.5$, which is seen in all of our results.

Figures 1 are for $U / t=20$. Figure 1(right panel) shows the full phase diagram in temperature versus chemical potential. Figures 1(left, middle panels) show the details in temperature versus electron density and chemical potential, respectively. It is seen that an antiferromagnetic phase occurs around half-filling, purely due to electron hopping, since the Hubbard Hamiltonian [Eq.(1)] does not contain an explicit antiferromagnetic coupling. In fact, we traced the occurrence of this antiferromagnetic phase to the non-zero value of the pair-breaking strength $t_{1}$. The antiferromagnetic phase is unstable to at most $10 \%$ hole or electron doping from half filling. Between 30 to $40 \%$ hole or electron doping, a $\tau$ phase occurs in which the vacancy hopping strength $t_{0}$ or the pair hopping strength $t_{2}$ [see Eq.(7)], respectively, renormalizes to infinity under repeated renormalization-group transformations. Thus, for hole doping, under repeated renormalization-group transformations, $t_{0} \rightarrow \infty, J / t_{0}=2, V_{2} / t_{0}=3 / 2, \mu / t_{0}=$ $6, t_{i \neq 0}=0, U \rightarrow \infty, t_{i} / U=0, V_{i} / U=0$. Symmetrically, for electron doping, the overbarred variables of Eq.(10) have this behavior. In all other regions of the phase diagram, all hopping strengths renormalize to zero under repeated renormalization-group transformations. Near the $\tau$ phase, a first-order phase transition (dotted curves) occurs, seen as a single curve in Fig.1 (center) in terms of electron chemical potential and opening up into a coexistence region in Fig.1 (left) in terms of electron density. The latter shows the distinctive feature of this first-order transition, namely that it involves a very narrow phase separation, e.g., a discontinuity in electron density of less than $2 \%$. This is similar to what is seen experimentally in lanthanide compounds. [8] At temperatures above the $\tau$ phase, a sequence of antiferromagnetic and disordered phases is seen, at many temperature scales [Figs.1,2 (center)]. We interpret this as the presence of an incommensurate spin modulation phase, with a temperature- and (less strongly, by the alignment of the sequencing) density-dependent periodicity. Our renormalization-group transformation, with a commensurate rescaling factor and a built-in approximation, acts as a spurious substrate potential which, at small incommensuration, registers the incommensurate phase and, at large incommensuration, disorders it. The incommensurate phase that we thus deduce is indicated in Figs.1,2 (left). The features described above were also seen in the simpler, less realistic, tJ model. [3, [4]

As $U / t$ is decreased, the first-order phase boundary moves with respect to the $\tau$ phase. It is seen that, for $U / t=4.44$ (Figs.2), it actually abuts the boundary of the $\tau$ phase and, for $U / t=0.8$ (Figs.3), it is on the other side of the $\tau$ phase.

We have thus calculated a finite-temperature phase diagram for the $d=3$ Hubbard model that is rich in phase transition phenomena. We have also repeated the same 
calculation for $d=2$. We find that no phase separation (in contrast to the tJ model [3, 4]) or other phase transition occurs at finite temperature for the Hubbard model [Eq.(1)] in $d=2$.

This research was supported by the Italian Istituto Nazionale di Fisica Nucleare (INFN), U.S. Department of Energy under Grant No. DE-FG02-92ER45473, and by the Scientific and Technical Research Council of Turkey (TÜBITAK). We gratefully acknowledge the hospitality of the Feza Gürsey Research Center for Basic Sciences and of the Istanbul Technical University.

[1] J. Hubbard, Proc. Royl Soc. A 276, 238 (1963); 277, 237 (1964); 281, 401 (1964).

[2] S.A. Kivelson, V.J. Emery, and H.Q. Lin, Phys. Rev. B 42, 6523 (1990).

[3] A. Falicov and A.N. Berker, Phys. Rev. B 51, 12458 (1995).

[4] A. Falicov and A.N. Berker, Turk. J. Phys. 19, 127 (1995).

[5] M. Suzuki and H. Takano, Phys. Lett. A 69, 426 (1979).

[6] H. Takano and M. Suzuki, J. Stat. Phys. 26, 635 (1981).

[7] G.S. Rushbrooke and P.J. Wood, Mol. Phys. 6, 409 (1963), calculate this effect as $14 \%$ from series expansion.

[8] F.C. Chou and D.C. Johnston, Phys. Rev. B 54, 572 (1996).

\section{Figure Captions}

Figs. 1: Calculated phase diagram of the $d=3$ Hubbard model for $U / t=20$. First- and second-order phase boundaries are shown with dotted and full curves, respectively. Fig.1(right panel) shows the full phase diagram, which is symmetric about $\mu / U=0.5$. Antiferromagnetic $[a]$, disordered $[D]$, and $\tau$ phases are seen. In the $\tau$ phases, the hopping strength $t_{0}$ or $t_{2}$ renormalizes to infinity, for hole or electron doping respectively. Above the $\tau$ phase, a sequence of antiferromagnetic and disordered phases [Fig.1(center panel)] is interpreted as an incommensurate spin modulation phase [Fig.1(left panel)]. As seen in (left), the first-order phase boundary has a very narrow coexistence region.

Figs. 2: Calculated phase diagram of the $d=3$ Hubbard model for $U / t=4.44$.

Figs. 3. Calculated phase diagram of the $d=3$ Hubbard model for $U / t=0.8$. 

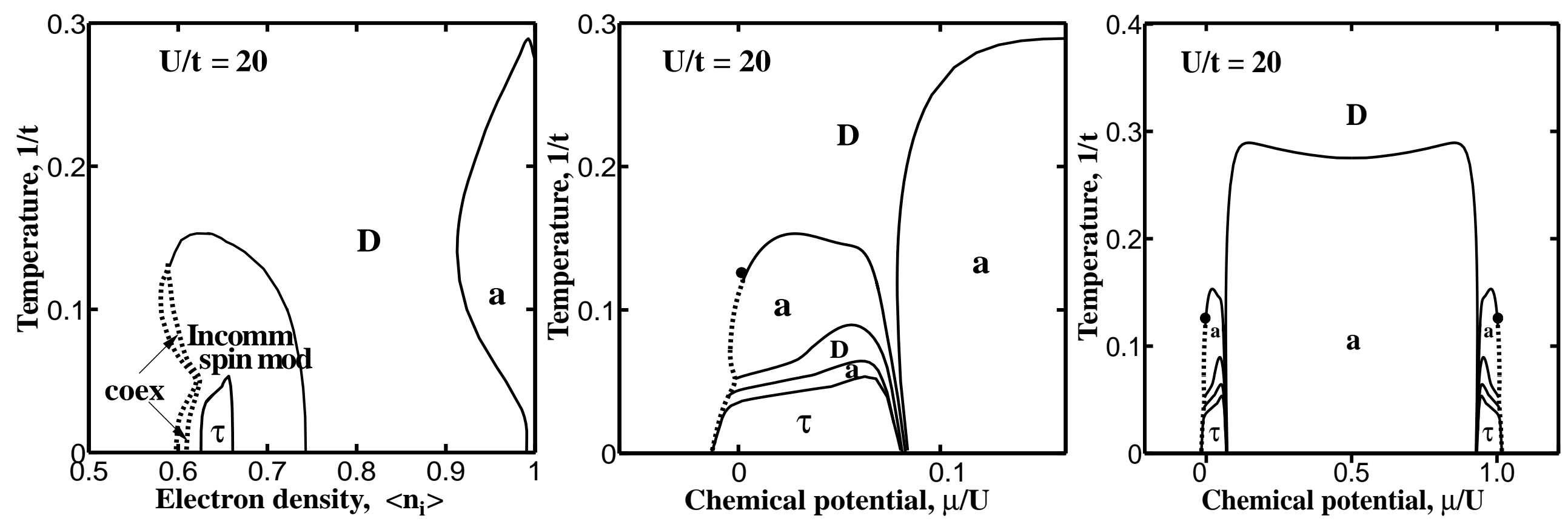

\section{Figure 1}



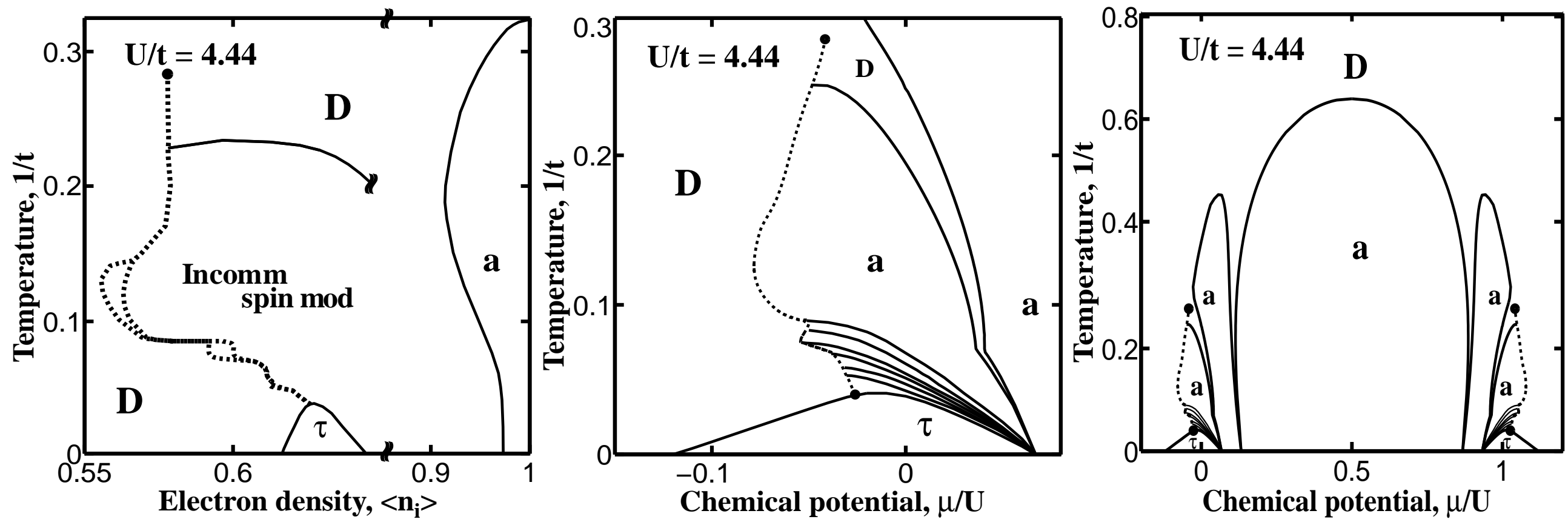

Figure 2 

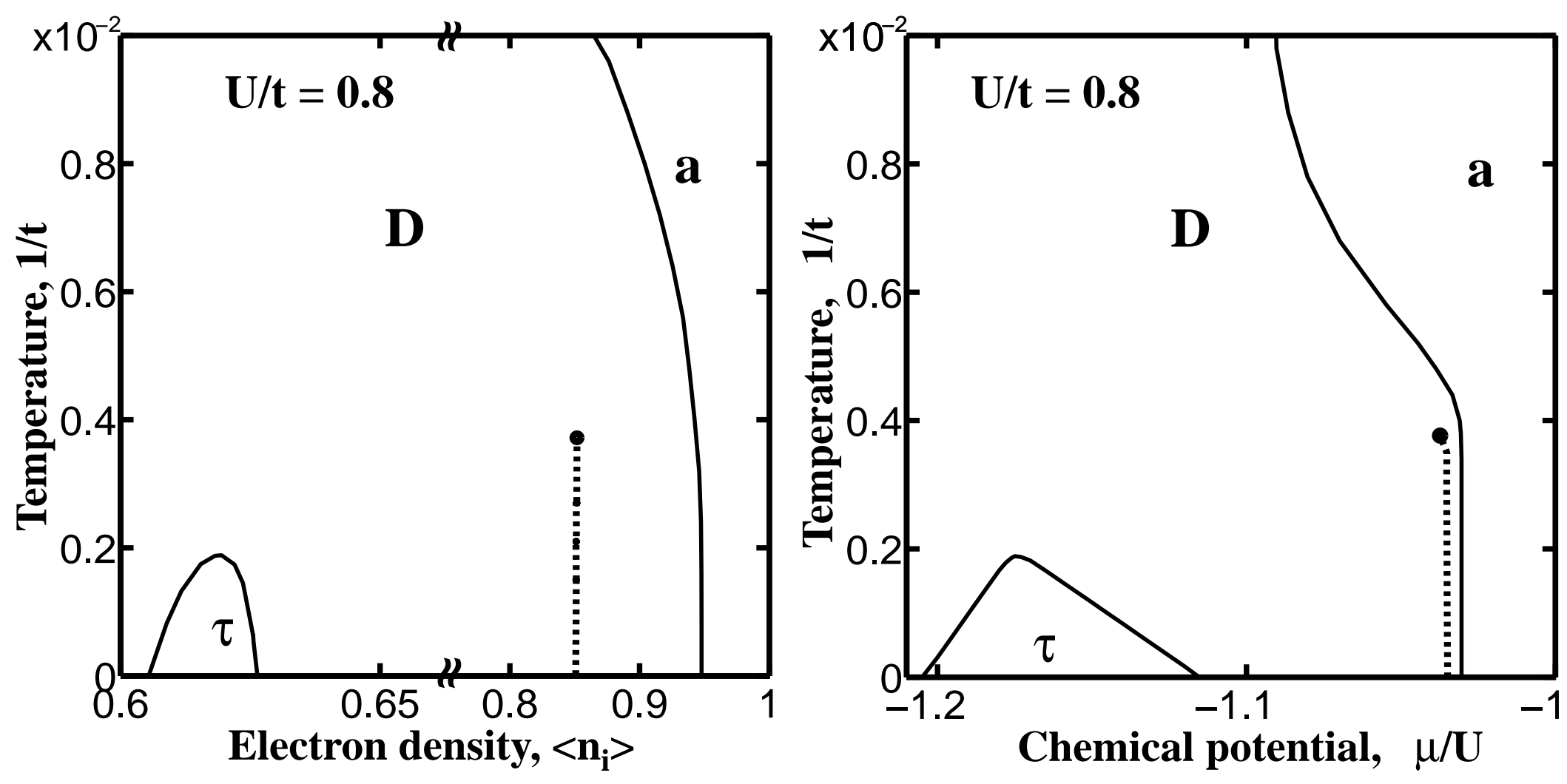

Figure 3 